\title{
Multicast OLSP Establishment Scheme in OVPN over IP/GMPLS over DWDM
}

\author{
Jeong-Mi Kim ${ }^{1}$, Oh-Han Kang ${ }^{2}$, Jae-Il Jung ${ }^{3}$, and Sung-Un Kim ${ }^{1, \star}$ \\ ${ }^{1}$ Pukyong National University, 599-1 Daeyeon 3-Dong Nam-Gu, Busan, \\ 608-737, Korea \\ kimjm@pknu.ac.kr, kimsu@pknu.ac.kr \\ ${ }^{2}$ Andong National University, 388 Song-chon Dong, Andong, Kyoungbuk, \\ 760-749, Korea \\ ohkang@andong.ac.kr \\ ${ }^{3}$ Hanyang University, 17 Haengdang-Dong Seongdong-Gu, Seoul, 133-791, Korea \\ jijung@hanyang.ac.kr
}

\begin{abstract}
OVPN (Optical Virtual Private Network) over IP (Internet Protocol)/GMPLS (Generalized Multi-Protocol Label Switching) over DWDM (Dense Wavelength Division Multiplexing) technology with QoS assurances is considered as a promising approach for the next generation OVPN. In this paper, we suggest a multicast OLSP (Optical Label Switched Path) establishment mechanism for supporting high bandwidth multicast services. For the establishment of the multicast OLSP, we propose a new multicast tree generation algorithm VS-MIMR (Virtual Source-based Minimum Interference Multicast Routing) that finds the minimum interference path between virtual source nodes. We also suggest an entire OVPN control mechanism to adapt the operation of the routing and signaling protocols of GMPLS.
\end{abstract}

\section{Introduction}

OVPNs are expected to be one of the major applications in the future optical networks. Therefore the OVPN over IP/GMPLS over DWDM technology has been suggested as a favorable approach for realizing the next generation VPN services [1].

In this paper, the characteristics of the OVPN multicast services are analyzed. And the establishment of multicast OLSP is investigated in two steps; OLSP establishment preparation phase and OLSP establishment phase. In the OLSP establishment phase, a new multicast tree generation algorithm, VS-MIMR is proposed. We also suggest an entire OVPN control mechanism to establish multicast OLSP adapting the operation of the routing and signaling protocols of GMPLS.

The rest of this paper is organized as follows. In section 2, we describe the functional architecture and operation of the QoS guaranteed OVPN. In Sections 3, we propose the multicast OLSP establishment scheme. In Section 4, the conclusion is presented.

\footnotetext{
^ Corresponding author.
} 


\section{Functional Architecture and Operation of QoS Guaranteed OVPN}

We propose the functional architecture for providing the optical QoS in OVPN as shown in Fig.1. For establishing the multicast OLSP, a Customer Agent requests a CE (Client Edge)-to-CE OLSP establishment with SLA(Service Level Agreement) parameters to the Negotiation Policy Agent. Once the Negotiation Policy Agent in an ingress PE (Provider Edge) receives a trigger for setting up an OLSP, it invokes the QoS Routing Policy Agent for routing and wavelength assignment with the QoS parameters extracted from the request.

Based on the OVPN membership and resource information gathered by OSPFTE+ (Open Shortest Path First with Traffic Engineering extensions) [2] and MPBGP (Multi-Protocol Border Gateway Protocol) [3, the OVPN Routing Agent calculates the QoS guaranteed tree for establishing the multicast OLSP. In this process, the VS-MIMR algorithm choosing a tree is proposed to calculate the QoS guaranteed multicast tree. After the tree calculation, the OVPN Signaling Agent in the control plane is invoked to reserve the optical resource with the GMPLS signaling protocol, the RSVP-TE+ (Resource ReSerVation Protocol with Traffic Engineering extensions) [4].

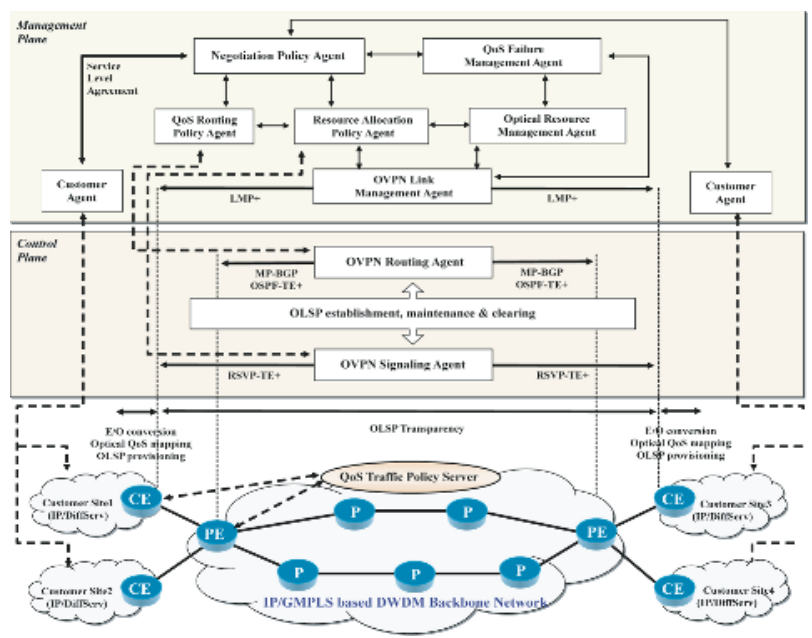

Fig. 1. The functional architecture of QoS guaranteed OVPN

\section{Multicast OLSP Establishment}

\subsection{Preparation Mechanism for OLSP Establishment}

Establishment of CE-to-CE Control Channel by LMP (Link Management Protocol): The control channels are used to exchange the control plane 
information such as the link provisioning, fault management information, label distribution information (implemented using a signaling protocol such as RSVP$\mathrm{TE}+$ ), and network topology, state distribution information (implemented using traffic engineering routing protocols such as OSPF-TE+).

The two core procedures of the LMP are the control channel management and link property correlation[5]. The control channel management is used to establish and maintain the control channels between the adjacent nodes. This is done by using the CONFIG and HELLO messages exchange. The link property correlation is used to synchronize the TE link properties and verify the TE link configuration.

Routing Information Exchange by OSPF-TE+: In this paper, we assume the routing information is distributed by OSPF-TE+ 2] between the PE nodes. The connection with the adjacent nodes is established by exchanging the Hello packet between the adjacent nodes. And then, only the LSA (Link State Advertisements) headers are exchanged and the recent needed information among them is checked through the Database Description packet. In the procedure of the database exchange, the recent needed information is requested through the Link State Request packet and the Link State Update packet containing the LSAs (Router-LSA, TE-LSA, and etc.) transmits the routing information.

Routing Information Exchange by MP-BGP: The MP-BGP is an extended BGP-4 protocol for the exchange of not only the IPv4 routing information but also the routing information of the diverse network layer protocols [3]. It is also used for the exchange of the membership information among the customer sites in the same OVPN. In the procedure of the neighbor connection, the adjacent relation is set with other nodes by using the OPEN message, and the negotiation of the related parameters (autonomous system number, version of BGP, BGP Router ID, and etc.) are exchanged. The routing information is exchanged by using a UPDATE message.

After forming such an entire routing table of the OVPN, the QoS guaranteed path is established through the SLA negotiation procedure at the time of a connection request. The appropriate OLSP is calculated by the mechanism explained in the next section.

\subsection{QoS Guaranteed Tree Establishment Mechanism}

SLA Negotiation for Multicast Session: In the case of the multicast OLSP establishment, the SLA negotiation procedure is also required between the OVPN backbone network and the customer site to establish the QoS guaranteed multicast tree.

When the QoS-TP (Traffic Policy) server (we assume this server is belong to the management plane in Fig.1) receives the SLA request that contains the multicast session information and the QoS parameters, it sends the VS_QUERY message to all the VSs in the OVPN network so that the PVS (Primary Virtual Source) and SVSs (Secondary Virtual Sources) can be found as shown in Fig.2. All the VSs respond to the QoS TP server with the VS_REPORT message. 


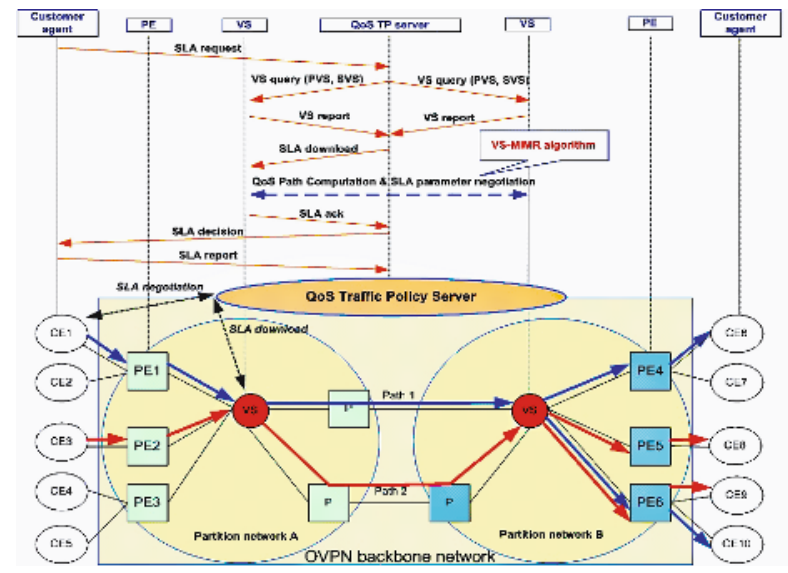

Fig. 2. SLA negotiation procedure for multicast service

When the QoS-TP server gets the information of all PVS and SVSs, it downloads the SLA parameters onto the Negotiation Policy Agent of the PVS in order to establish the connections to all the SVS nodes. At this time, using the VS-MIMR algorithm, we improve the resource utilization in the OVPN backbone network.

VS-MIMR for Multicast Tree Generation: The VS-MIMR algorithm is suggested to choose minimum interference paths. The proposed algorithm overcomes the limitation of VS-based method [6] and provides an efficient use of wavelengths.

Fig.2 illustrates the VS-MIMR algorithm. There are two potential sourcedestinations pairs such as (PE1, PE4\&PE6) and (PE2, PE5\&PE6). When Path 1 is chosen for the first multicast session in order to make a resource reservation for the path between a PVS-SVS pair, the other multicast session may share the same path having a minimum-hop path. It can lead high blocking probability by inefficiently using the resource due to the traffic concentration on that path. Thus, it is better to pick Path 2 that has a minimum interference effect for other future multicast session requests even though the path is longer than Path 1. We define that a segment means a path between VS nodes. And each segment must follow the wavelength continuity constraint, because only VS nodes can have a wavelength splitting and conversion capability. We define some additional notations used in this algorithm as follows.

- $G(N, L, W)$ : The given network, where $N$ is the set of nodes, $L$ is the set of links, and $W$ is the set of wavelengths per link.

- $\left(v_{p}, v_{s}\right)$ : A PVS-SVS node pair.

- $(a, b)$ : A PVS-SVS node pair for current requests, where $(a, b) \in\left(v_{p}, v_{s}\right)$.

- $\Lambda$ : The set of potential PVS-SVS node pairs that can be requested by multicast session in the future, where $\forall\left(v_{p}, v_{s}\right) \in \Lambda$

- $S_{p s}^{n}$ :The $n$th segment of the set of minimum hop segments connecting the path.

- $C_{p s}$ : The set of critical links between the $\left(v_{p}, v_{s}\right)$ pair, that is, $C_{p s}$ are shared with other node pairs at the same time. 
- $F_{p s}$ : The number of available wavelengths on bottleneck segment that has the smallest residual wavelengths.

- $R\left(S_{p s}^{n}\right)$ : The number of residual wavelengths on the segment $S_{p s}^{n}$.

- $\alpha_{p s}$ : The weight for a segment according to the degree of multicast session resource reservation requests between the PVS node and SVS node.

- $w\left(S_{p s}^{n}\right)$ : The accumulated total weights for $S_{p s}^{n}$.

- $\Delta$ : A threshold value of available wavelengths on $S_{p s}^{n}$ (30\% of the total wavelengths in $\left.S_{p s}^{n}\right)$.

Based on these notations, the segment weights are determined as follow:

$$
\begin{gathered}
\operatorname{Max} \sum \alpha_{p s} \cdot F_{p s} . \\
W\left(S_{p s}^{n}:\left(S_{p s}^{n}: e \in C_{p s}\right) \cap\left(R\left(S_{p s}^{n}\right)<\triangle\right) .\right. \\
\sum_{\forall\left(v_{p}, v_{s}\right) \in \Lambda \backslash(a, b)} \alpha_{p s}\left(\partial F_{p s} / \partial R\left(S_{p s}^{n}\right)\right) . \\
\left\{\begin{array}{l}
\partial F_{p s} / \partial R\left(S_{p s}^{n}\right)=1\left[i f\left(v_{p}, v_{s}\right): S_{p s}^{n} \in C P_{p s}\right] \\
\partial F_{p s} / \partial R\left(S_{p s}^{n}\right)=0[\text { otherwise }]
\end{array}\right. \\
w\left(S_{p s}^{n}\right)=\sum_{\left(v_{p}, v_{s}\right): S_{p s}^{n} \in C P_{p s}} \alpha_{p s} .
\end{gathered}
$$

Equation (1) represents the minimum interference of the wavelength path decision between the PVS node and SVS node. Equation (2) determines the $C P$ (congestion path) with congestion possibility for potential future connection requests between the VS nodes.

We presents the weight of each segment for all $\left(v_{p}, v_{s}\right)$-pairs in the set $\Lambda$ except the current request when setting up a connection as shown in equation (3). And equation (4) allocates the differentiated values to the $n$th segment between the VS nodes. Calculating the weight of all nodes is difficult, so we apply equation (4) to equation (3). Finally, computing the segment weights is simplified as shown in equation (5). Therefore, the VS-MIMR decides a wavelength path that has a minimum value of segment weight $w\left(S_{p s}^{n}\right)$.

\subsection{Multicasting Distribution Tree Construction Using RSVP-TE+}

After the tree calculation, a point-to-multipoint OLSP tree (P2MP tunnel) must be constructed by the RSVP-TE+ extensions for multicasting services. Although the P2MP OLSP is constituted of the multiple source-to-one leaf (S2L) subOLSPs, we can signal all S2L sub-OLSPs in one PATH message with the EXPLICIT_ROUTE object (ERO), P2MP SECONDARY_EXPLICIT_ROUTE object (SERO), and S2L_SUB_LSP object (S2LO) [7]. Fig.3 shows a P2MP OLSP with PE1 as a source node and three destination nodes (PE2, PE3 and PE4). When the branch nodes (VS1 and VS2) receive the PATH message, it generates the multiple PATH messages with the different EROs and SEROs. After sending out the PATH messages to all nodes of the multicasting tree, this will be confirmed by the RESV message with the ROUTE_RECORD object (RRO) 


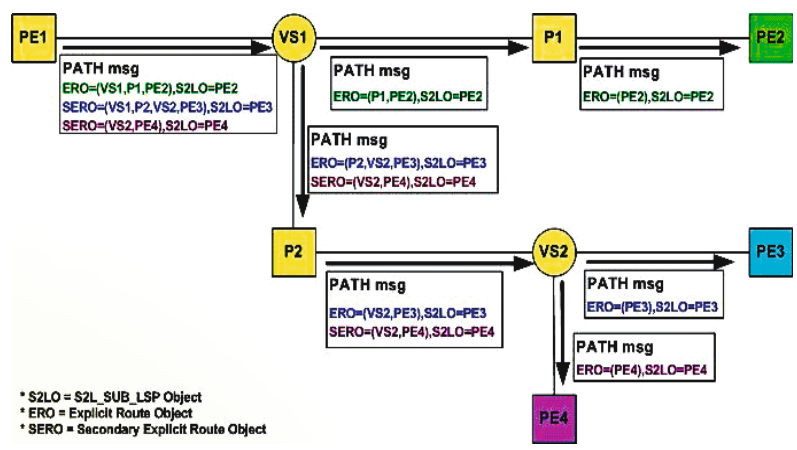

Fig. 3. Multicasting distribution tree construction

and P2MP SECONDARY_ROUTE_RECORD objects (SRROs) at each link of the multicasting tree[7.

\section{Conclusion}

In this paper, the functional architecture and the interoperation of the control plane and management plane of the QoS guaranteed OVPN are proposed. For the establishment of the multicast OLSP, we propose the VS-MIMR algorithm that finds the minimum interference path between virtual source nodes. We also suggest an entire OVPN control mechanism to adapt the operation of the routing and signaling protocols of GMPLS.

\section{Acknowledgment}

This work was supported by grant No.(R01-2003-000-10526-0) from Korea Science \& Engineering Foundation.

\section{References}

1. Z. Zhang, et al., An Overview of Virtual Private Network (VPN): IP VPN and Optical VPN, Photonic Network Communications, vol.7, no.3, pp.213-225, 2004.

2. K. Kompella and Y. Rekhter, OSPF Extensions in support of Generalized MultiProtocol Label Switching, IETF RFC 4203, Oct. 2005.

3. T. Bates et al, Multiprotocol Extension for BGP-4, RFC2858, June 2000.

4. L. Berger, GMPLS Signaling Resource ReserVation Protocol-Traffic Engineering (RSVP-TE) Extensions, IETF RFC 3473, Jan. 2003.

5. J. Lang, Link Management Protocol (LMP), IETF RFC4204, Oct. 2005.

6. N. Sreenath et al., Virtual Source Based Multicast Routing in WDM Optical Networks, Photonic Network Communications, vol.3, no.3, pp.217-230, 2001.

7. R. Aggarwal et al., Extensions to RSVP-TE for Point to Multipoint TE LSPs, draft-ietf-mpls-rsvp-te-p2mp-01.txt, IETF Internet Draft, June 2005. 